\title{
Renin-angiotensin system inhibitor is associated with the reduced risk of all-cause mortality in COVID-19 among patients with/without hypertension
}

Huai-yu Wang ${ }^{1, *}$, Suyuan Peng ${ }^{1, *}$, Zhanghui $\mathrm{Ye}^{2}$, Pengfei $\mathrm{Li}^{2}$, Qing $\mathrm{Li}^{2}$, Xuanyu Shi ${ }^{1}$, Rui Zeng ${ }^{3}$, Ying Yao ${ }^{4}, \mathrm{Fan} \mathrm{He}^{3}$, Junhua $\mathrm{Li}^{3}$, Liu $\mathrm{Liu}^{3}$, Shuwang $\mathrm{Ge}^{3}$, Xianjun $\mathrm{Ke}^{5}$, Zhibin Zhou ${ }^{5}$, Gang $\mathrm{Xu}^{3}$, Ming-hui Zhao ${ }^{7,8}$, Haibo Wang $^{6}$, Luxia Zhang $(\bowtie)^{1,2,7}$, Erdan Dong ${ }^{9}$

${ }^{I}$ National Institute of Health Data Science at Peking University, Beijing 100191, China; ${ }^{2}$ Peking University Advanced Institute of Information Technology, Hangzhou 311215, China; ${ }^{3}$ Department of Nephrology, Tongji Hospital, Tongji Medical College, Huazhong University of Science and Technology, Wuhan 430030, China; ${ }^{4}$ Department of Clinical Nutrition, Tongji Hospital, Tongji Medical College, Huazhong University of Science and Technology, Wuhan 430030, China; ${ }^{5}$ Taikang Tongji (Wuhan) Hospital, Wuhan 430050, China; ${ }^{6}$ First Affiliated Hospital of Sun Yat-sen University, Guangzhou 510080, China; ${ }^{7}$ Renal Division, Department of Medicine, Peking University First Hospital, Peking University Institute of Nephrology, Beijing 100034, China; ${ }^{8}$ Peking-Tsinghua Center for Life Sciences, Beijing 100871, China; ${ }^{9}$ Department of Cardiology and Institute of Vascular Medicine, Peking University Third Hospital; Key Laboratory of Cardiovascular Molecular Biology and Regulatory Peptides, Ministry of Health; Key Laboratory of Molecular Cardiovascular Sciences, Ministry of Education; Beijing Key Laboratory of Cardiovascular Receptors Research, Beijing 100191, China

(C) Higher Education Press 2021

Abstract Consecutively hospitalized patients with confirmed coronavirus disease 2019 (COVID-19) in Wuhan, China were retrospectively enrolled from January 2020 to March 2020 to investigate the association between the use of renin-angiotensin system inhibitor (RAS-I) and the outcome of this disease. Associations between the use of RAS-I (angiotensin-converting enzyme inhibitor (ACEI) or angiotensin receptor blocker (ARB)), ACEI, and ARB and in-hospital mortality were analyzed using multivariate Cox proportional hazards regression models in overall and subgroup of hypertension status. A total of 2771 patients with COVID-19 were included, with moderate and severe cases accounting for $45.0 \%$ and $36.5 \%$, respectively. A total of $195(7.0 \%)$ patients died. RAS-I (hazard ratio $(H R)=0.499,95 \%$ confidence interval $(C I) 0.325-0.767)$ and $A R B(H R=0.410,95 \%$ CI $0.240-0.700)$ use was associated with a reduced risk of all-cause mortality among patients with COVID-19. For patients with hypertension, RAS-I and ARB applications were also associated with a reduced risk of mortality with HR of 0.352 (95\% CI 0.162-0.764) and 0.279 (95\% CI 0.115-0.677), respectively. RAS-I exhibited protective effects on the survival outcome of COVID-19. ARB use was associated with a reduced risk of all-cause mortality among patients with COVID-19.

Keywords COVID-19; RAS inhibitor; hypertension; all-cause mortality

\section{Introduction}

Coronavirus disease 2019 (COVID-19) is a global pandemic caused by the severe acute respiratory syndrome coronavirus 2 (SARS-CoV-2) [1,2]. Angiotensin-converting enzyme 2 (ACE2) serves as the target receptor of SARS-CoV-2 by facilitating virus entry and replication in

Received August 11, 2020; accepted February 20, 2021

Correspondence: Luxia Zhang, zhanglx@bjmu.edu.cn

\footnotetext{
*Huai-yu Wang and Suyuan Peng equally contributed to this study.
}

host cells, such as alveolar epithelial cells [3,4]. ACE2 is an essential component of the renin-angiotensin system (RAS), a major hormonal system regulating physiological homeostasis through multiple pathways including hemodynamics, electrolyte balance, vasomotion, inflammation, and tissue fibrosis $[3,5,6]$. Studies on SARS-CoV (pathogen of SARS, sharing $76.3 \%$ homology of amino acid sequence with SARS-CoV-2) found that injecting the binding domain of SARS-CoV (similar to that of SARSCoV-2) into mice exacerbates lung injury, which could be attenuated by blocking RAS and is strongly associated with ACE2 expression [3,7]. Despite the evidence on its 


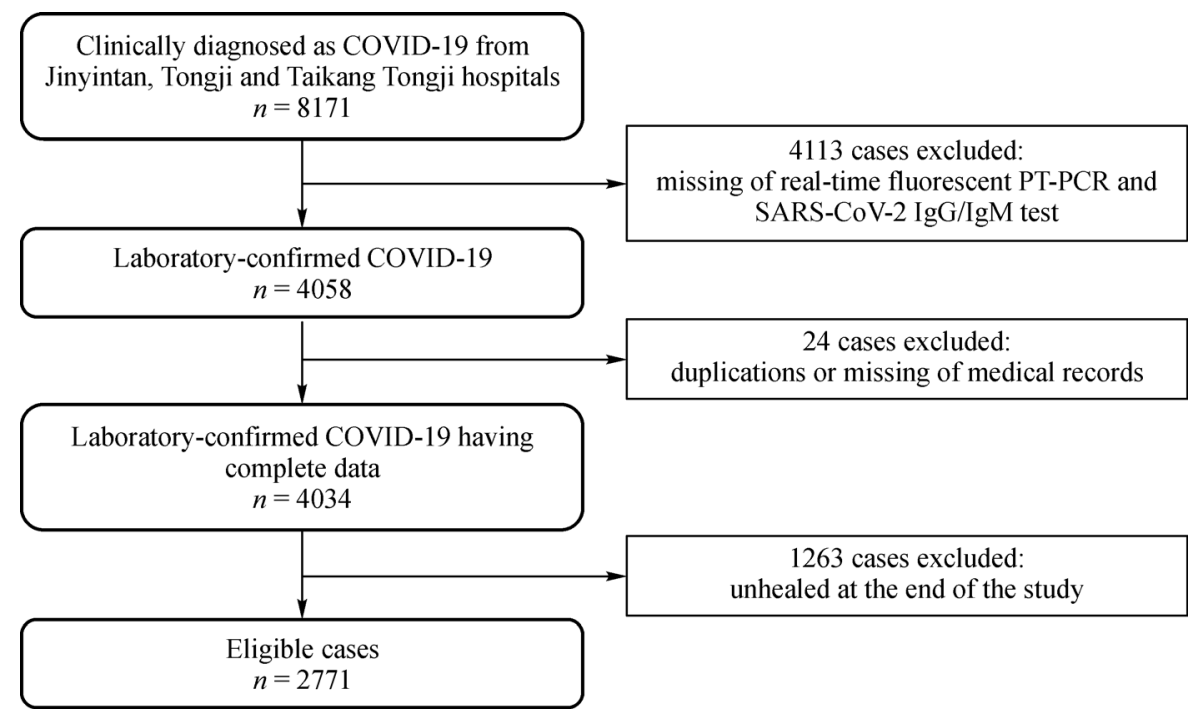

Fig. 1 Flowchart of enrollment process.

role as a virus-target co-receptor, its physiologically protective effects on RAS, and experimental indication in SARS, RAS inhibitor (RAS-I) administration among patients with COVID-19 remains controversial [8].

RAS-Is, including angiotensin-converting enzyme inhibitor (ACEI) and angiotensin receptor blocker (ARB), are routinely administered to patients with hypertension, diabetes, kidney diseases, and several types of cardiovascular diseases. Evidence on the association between RAS-I and COVID-19 outcome is clinically relevant for the treatment of patients with COVID-19. Several studies investigated the association between RAS-I use and COVID-19 outcome, but the results were inconsistent [9-13]. Li et al. found no substantial difference in the severity and outcomes of 1178 patients with COVID-19 and hypertension who received RAS-I [9]. Mehta et al. enrolled 18472 participants receiving COVID-19 tests and showed that RAS-I use was not associated with COVID-19 risk; however, the correlation of this treatment with the risk of hospital admission and intensive care unit (ICU) admission was observed among 1735 patients with COVID-19. A high mortality was reported among RAS-I users compared with that among non-RAS-I users (3.8\% vs. 2.1\%) [13]. Xu et al. investigated the effects of RAS-I administration among 702 patients with COVID-19 and reported no remarkable association between RAS-I use and disease outcomes [14]. The inconsistency of these results might be caused by the heterogeneous population, various sample size, different documentation techniques for RAS-I use, and involvement of potential confounders. Further studies using large sample size are needed.

This study investigated the association between RAS-I use and COVID-19 outcome in a large sample of patients with COVID-19 from Wuhan, China.

\section{Materials and methods}

\section{Study population}

The National Health Commission of China collected COVID-19-related medical records from the designated hospitals in China to provide scientific evidence on medical treatment for patients with COVID-19. The standardized in-patient discharge summaries and the electronic full medical records of confirmed COVID-19 cases were submitted to the COVID-19 reporting system of National Health Commission of China. This retrospective analysis was based on the national database of the COVID19 reporting system. The beginning and the cutoff dates for data gathering were January 1, 2020 and March 23, 2020, respectively. Participant enrollment process is shown in Fig. 1.

All data, including demographic characteristics, comorbidities, laboratory tests, treatments, morbidities, and survival status, were extracted from the data sets. Two researchers independently reviewed the collected data and extraction forms for quality control purposes.

COVID-19 was diagnosed according to the Diagnosis and Treatment Protocol for COVID-19 (Trial Version 7) issued by the National Health Commission of China on March 3, 2020 [15]. Patients with positive result of realtime fluorescence PT-PCR or SARS-CoV-2 IgG/IgM test were regarded as confirmed cases with COVID-19.

Disease severity was evaluated according to the above protocol [15]. Mild cases were identified as having mild clinical symptoms without any sign of pneumonia on CT scanning. Moderate cases were radiologically defined as having pneumonia, fever, and respiratory symptoms. Severe cases were defined as meeting any of the following 
Table 1 Demographic characteristics

\begin{tabular}{|c|c|c|c|c|}
\hline \multirow{2}{*}{ Factors } & \multirow{2}{*}{$\begin{array}{l}\text { Overall } \\
(n=2771)\end{array}$} & \multicolumn{2}{|c|}{ RAS-I administration } & \multirow{2}{*}{$P$ value } \\
\hline & & Yes $(n=280)$ & No $(n=2491)$ & \\
\hline Age, $n(\%)$ & & & & $<0.001$ \\
\hline$<18$ years & $16(0.6 \%)$ & $0(0.0 \%)$ & $16(0.6 \%)$ & \\
\hline $18-44$ years & $477(17.2 \%)$ & $12(4.3 \%)$ & $465(18.7 \%)$ & \\
\hline $45-64$ years & $1262(45.5 \%)$ & $113(40.4 \%)$ & $1149(46.1 \%)$ & \\
\hline$\geqslant 65$ years & $1016(36.7 \%)$ & $155(55.4 \%)$ & $861(34.6 \%)$ & \\
\hline Male, $n(\%)$ & $1328(47.9 \%)$ & $147(52.5 \%)$ & $1181(47.4 \%)$ & 0.110 \\
\hline \multicolumn{5}{|l|}{ Comorbidity, $n(\%)$} \\
\hline Hypertension & $590(21.5 \%)$ & $141(50.4 \%)$ & $449(18.2 \%)$ & $<0.001$ \\
\hline Diabetes & $299(10.9 \%)$ & $56(20.0 \%)$ & $243(9.8 \%)$ & $<0.001$ \\
\hline Cardiovascular disease & $133(4.8 \%)$ & $31(11.1 \%)$ & $102(4.1 \%)$ & $<0.001$ \\
\hline Chronic kidney disease & $26(0.9 \%)$ & $4(1.4 \%)$ & $22(0.9 \%)$ & 0.330 \\
\hline Chronic obstructive pulmonary disease & $56(2.0 \%)$ & $5(1.8 \%)$ & $51(2.1 \%)$ & 0.750 \\
\hline Cancer & $30(1.1 \%)$ & $6(2.1 \%)$ & $24(1.0 \%)$ & 0.120 \\
\hline None of above & $1947(70.3 \%)$ & $128(45.7 \%)$ & $1819(73.0 \%)$ & $<0.001$ \\
\hline \multicolumn{5}{|l|}{ Laboratory test } \\
\hline WBC $\left(\times 10^{9}\right.$ cells $\left./ \mathrm{L}\right)$ & $6.8(5.4,8.9)$ & $7.9(5.6,13.2)$ & $6.8(5.4,8.8)$ & 0.007 \\
\hline $\mathrm{LYM}\left(\times 10^{9}\right.$ cells $\left./ \mathrm{L}\right)$ & $1.2(0.7,1.6)$ & $0.9(0.4,1.3)$ & $1.2(0.7,1.6)$ & $<0.001$ \\
\hline hs-CRP (mg/L) & $19.9(2.4,78.4)$ & $65.7(5.0,161.6)$ & $19.3(2.3,75.6)$ & $<0.001$ \\
\hline $\mathrm{PCT}(\mathrm{ng} / \mathrm{L})$ & $0.07(0.04,0.21)$ & $0.14(0.05,0.61)$ & $0.07(0.04,0.21)$ & 0.003 \\
\hline IL-6 (pg/mL) & $6.3(2.1,14.4)$ & $12.7(4.0,72.6)$ & $6.3(2.1,14.2)$ & $<0.001$ \\
\hline hs-cTn (ng/mL) & $4.5(1.9,11.7)$ & $11.5(4.7,29.6)$ & $4.4(1.9,11.4)$ & $<0.001$ \\
\hline $\mathrm{SCr}(\mu \mathrm{mol} / \mathrm{L})$ & $70.0(58.0,86.0)$ & $88.4(71.0,133.0)$ & $70.0(58.0,85.6)$ & $<0.001$ \\
\hline SBP (mmHg) & $124.4(116.7,132.5)$ & $119.5(71.9,135.6)$ & $124.4(116.8,132.5)$ & 0.087 \\
\hline DBP (mmHg) & $75.4(71.1,80.7)$ & $86.2(74.7,117.3)$ & $75.2(71.0,80.5)$ & $<0.001$ \\
\hline \multicolumn{5}{|l|}{ Complication, $n(\%)$} \\
\hline Acute respiratory distress syndrome & $416(15.0 \%)$ & $68(24.3 \%)$ & $348(14.0 \%)$ & $<0.001$ \\
\hline Heart failure & $296(10.7 \%)$ & $59(21.1 \%)$ & $237(9.5 \%)$ & $<0.001$ \\
\hline Acute myocardial infarction & $250(9.0 \%)$ & $41(14.6 \%)$ & $209(8.4 \%)$ & $<0.001$ \\
\hline Acute kidney injury & $107(3.9 \%)$ & $22(7.9 \%)$ & $85(3.4 \%)$ & $<0.001$ \\
\hline Multiple organ dysfunction syndrome & $265(9.6 \%)$ & $52(18.6 \%)$ & $213(8.6 \%)$ & $<0.001$ \\
\hline \multicolumn{5}{|l|}{ Treatment, $n(\%)$} \\
\hline Anti-virus drug & $1861(67.2 \%)$ & $198(70.7 \%)$ & $1663(66.8 \%)$ & 0.180 \\
\hline Glucocorticoid & $625(22.6 \%)$ & $73(26.1 \%)$ & $552(22.2 \%)$ & 0.140 \\
\hline Chloroquine/hydroxychloroquine & $293(10.6 \%)$ & $37(13.2 \%)$ & $256(10.3 \%)$ & 0.130 \\
\hline Tocilizumab & $41(1.5 \%)$ & $10(3.6 \%)$ & $31(1.2 \%)$ & 0.002 \\
\hline NPPV & $316(11.4 \%)$ & $49(17.5 \%)$ & $267(10.7 \%)$ & $<0.001$ \\
\hline IPPV & $129(4.7 \%)$ & $19(6.8 \%)$ & $110(4.4 \%)$ & 0.074 \\
\hline CRRT & $51(1.8 \%)$ & $15(5.4 \%)$ & $36(1.4 \%)$ & $<0.001$ \\
\hline Severity, $n(\%)$ & & & & $<0.001$ \\
\hline Mild cases & $109(3.9 \%)$ & $5(1.8 \%)$ & $104(4.2 \%)$ & \\
\hline Moderate cases & $1247(45.0 \%)$ & $96(34.3 \%)$ & $1151(46.2 \%)$ & \\
\hline Severe cases & $1012(36.5 \%)$ & $119(42.5 \%)$ & $893(35.8 \%)$ & \\
\hline Critical & $403(14.5 \%)$ & $60(21.4 \%)$ & $343(13.8 \%)$ & \\
\hline Outcome, $n(\%)$ & & & & 0.072 \\
\hline Recovery & $2576(93.0 \%)$ & $253(90.4 \%)$ & $2323(93.3 \%)$ & \\
\hline Death & $195(7.0 \%)$ & $27(9.6 \%)$ & $168(6.7 \%)$ & \\
\hline
\end{tabular}

Abbreviations: RAS-I, renin-angiotensin system inhibitor; WBC, while blood cell; LYM, lymphocyte count; hs-CRP, hyper-sensitive C-reactive protein; PCT, procalcitonin; IL-6, interleukin-6; hs-cTn, hyper-sensitive cardiac troponin; SCr, serum creatinine; SBP, systolic blood pressure; DBP, diastolic blood pressure; NPPV, noninvasive positive pressure mechanical ventilation; IPPV, invasive positive pressure mechanical ventilation; CRRT, continuous renal replacement therapy. 
criteria: (1) respiratory distress (respiratory rate $\geqslant 30$ count $/ \mathrm{min}$ ); (2) oxygen saturation $\leqslant 93 \%$ at rest; and (3) arterial partial pressure of oxygen $\left(\mathrm{PaO}_{2}\right)$ /fraction of inspired oxygen $\left(\mathrm{FiO}_{2}\right) \leqslant 300 \mathrm{mmHg}$. Critical cases were defined as experiencing respiratory failure and requiring mechanical ventilation, shock, or other organ failures that requires intensive care unit (ICU) care.

This study was conducted with the authorization of National Health Commission of China and was approved by the Ethics Committee of Peking University Health Science Center (IRB00001052-20032) .

\section{RAS-I use and hypertension}

ACEI (including benazepril, imidapril, captopril, perindopril, enalapril, and fosinopril) and ARB (including valsartan, irbesartan, losartan, candesartan, olmesartan, allisartan, and telmisartan) administrations were documented in the electronic medical records during hospitalization. The use of any kind of ACEI or ARB during hospitalization were defined as RAS-I administration.

\section{Comorbidities and complications}

Comorbidities including hypertension, diabetes, coronary heart disease (CHD), chronic obstructive pulmonary disease (COPD), chronic kidney disease (CKD), and cancer were recorded. Hypertension was defined as: (1) with the diagnosis of hypertension in the medical records with ICD-10 codes I10-I15; (2) receiving antihypertensive therapy; and (3) systolic blood pressure (SBP)/diastolic blood pressure (DBP) $>140 / 90 \mathrm{mmHg}$. Diabetes was defined as: (1) with the diagnosis of diabetes in the medical records with ICD-10 codes E11-E14; (2) receiving hypoglycemic therapy; (3) HbAlc $\geqslant 6.5 \%$; and (4) fasting plasma glucose $\geqslant 7.0 \mathrm{mmol} / \mathrm{L}$. CHD (ICD10 code: I25.9), COPD (ICD-10 code: J44), and CKD (ICD-10 code: N00- N19, N25) were defined based on the diagnosis criteria in medical records.

Complications including acute respiratory distress syndrome (ARDS; ICD-10 codes: J80), heart failure (ICD-10 codes: I13.0, I13.2, I25.5, I43.8, I50.0, and I51.5), acute myocardial infarction (AMI; ICD-10 codes: I21, I22.8), acute kidney injury (AKI; ICD-10 codes: N17), and multiple organ dysfunction syndrome (MODS) were also analyzed and defined according to the diagnosis in the medical records based on related ICD-10 codes or respective criteria as follows. (1) ARDS was defined according to the Berlin definition [16]. (2) Heart failure was defined as the clinical syndrome characterized by typical symptoms (e.g., breathlessness, ankle swelling, and fatigue) that may be accompanied by signs (e.g., elevated jugular venous pressure, pulmonary crackles and peripheral edema) caused by a structural and/or functional cardiac abnormality [17]. (3) AMI was defined as hyper- sensitive cardiac troponin (hs-cTn) $>$ 99th percentile of normal reference values in the absence of any clinical features of myocardial ischemia [18]. (4) AKI was defined according to KDIGO clinical guidelines [19]. (5) MODS was defined as the progressive, potentially reversible dysfunction of two or more organ systems following acute, life-threatening disruption of systemic homeostasis.

\section{Other variables}

Information was collected regarding demographic characteristics, medications including anti-virus treatment, glucocorticoid, chloroquine/hydroxychloroquine and tocilizumab, mechanical ventilation including noninvasive positive pressure mechanical ventilation (NPPV) and invasive positive pressure mechanical ventilation (IPPV), and continuous renal replacement therapy (CRRT).

Laboratory tests included white blood cell (WBC, $\times$ $10^{9}$ cells $\left./ \mathrm{L}\right)$, lymphocyte count $\left(\mathrm{LYM}, \times 10^{9}\right.$ cells $\left./ \mathrm{L}\right)$, hyper-sensitive C-reactive protein (hs-CRP, mg/L), procalcitonin (PCT, ng/L), interleukin-6 (IL-6, pg/mL), hypersensitive cardiac troponin (hs-cTn, $\mathrm{ng} / \mathrm{mL}$ ), and serum creatinine $(\mathrm{SCr}, \pm \mathrm{mol} / \mathrm{L})$.

\section{Statistical methods}

Demographic characteristics, comorbidities, laboratory tests, treatments, complications, and outcomes were compared according to RAS-I administration. Normal and skewed distribution variables were presented by mean \pm standard deviation (SD) and median (interquartile range, IQR), respectively. Categorical variables were presented as proportions. Student's $t$ test, Mann-Whitney test, and Chi-square test were applied to compare the normally distributed, skewed distributed, and categorical variables between RAS-I administration groups, respectively. Subgroup analyses were conducted among patients with hypertension.

Cox proportional hazards regression models were applied to evaluate the association between RAS-I administration and all-cause mortality among patients with COVID-19. The results were presented as hazard ratio (HR) and 95\% confident interval (CI). Covariates including age (continuous), gender (female vs. male), comorbidities (hypertension, diabetes, CHD; yes or no), lymphocyte count (continuous), treatments (anti-virus drugs, glucocorticoid, tocilizumab, chloroquine/hydroxychloroquine; yes or no), and complications (ARDS, heart failure, AMI, AKI; yes or no) were included in these models. Subgroup survival analyses were performed among patients with hypertension. The proportional hazard assumption was fulfilled for all analyses tested using the time-dependent covariate method [20].

All statistical analyses were two-tailed, and $P$ value $<0.05$ indicated statistical significance. All analyses were 
conducted using Stata version 14.0 (Stata Corp LP, College Station, TX, USA).

\section{Results}

\section{Demographic and clinical characteristics}

A total of 4058 patients with COVID-19 with confirmed real-time fluorescent PT-PCR or SARS-CoV-2 IgG/IgM test were admitted to Jinyintan, Tongji and Taikang Tongji hospitals, Wuhan, China from January 1, 2020 to March 23, 2020. Among these patients, 1263 were not discharged at the end of the study, and 24 had incomplete data of medical records and thus were excluded in the present analyses. Finally, 2771 patients were eligible (Fig. 1) .The majority of the studied population aged 45 years or older $(82.2 \%)$, and $47.9 \%$ of them were males. Most patients were labeled as moderate $(45.0 \%)$ and severe $(36.5 \%)$ cases. Hypertension was the most common comorbidity $(n=590,21.5 \%)$. A total of $416(15.0 \%)$ patients developed ARDS, and 316 (11.4\%) and 129 (4.7\%) patients received NPPV and IPPV, respectively. The median duration of follow-up was $20(12,29)$ days. At the end of the study period, $2576(93.0 \%)$ patients recovered, and 195 (7.0\%) patients died.

A total of 280 patients received RAS-I during hospitalization, and $50.4 \%$ of them had hypertension. Among these patients, 43 (15.4\%) used ACEI only, 225 (80.4\%) used ARB only, and 12 (4.3\%) used ACEI and
ARB. Compared with the patients who did not receive RAS-I, those who received RAS-I were older and had higher proportion of comorbidities (hypertension: 50.4\% vs. $18.2 \%$; diabetes: $20.0 \%$ vs. $9.8 \%$; CHD: $11.1 \%$ vs. $4.1 \%$ ) and complications including ARDS, heart failure, AMI, AKI, and MODS $(P<0.001)$ (Table 1$)$.

\section{RAS-I administration and all-cause mortality}

A total of 195 patients died during hospitalization, 27 of them received RAS-I (Table 1). After the adjustment for potential confounders, Cox proportional hazards regression models showed that RAS-I $(\mathrm{HR}=0.499,95 \% \mathrm{CI}$ $0.325-0.767)$ and ARB $(\mathrm{HR}=0.410,95 \%$ CI 0.240 0.700 ) administrations were associated with a reduced risk of all-cause mortality among patients with COVID-19. However, no significant association was found between ACEI use and all-cause mortality $(\mathrm{HR}=0.892,95 \% \mathrm{CI}$ 0.473-1.682) (Table 2) .

\section{Subgroup analyses among patients with hypertension}

Among the 590 patients with hypertension, 141 (23.9\%) received RAS-I with the predominance of ARB use $(n=116,82.3 \%)$, and $64(10.8 \%)$ died during hospitalization. The mortality of patients receiving RAS-I was lower than that of patients not receiving RAS-I (5.7\% vs. $12.5 \%)$ (Table 3).

RAS-I $(H R=0.352,95 \%$ CI $0.162-0.764)$ and ARB $(\mathrm{HR}=0.279,95 \%$ CI $0.115-0.677)$ applications were

Table 2 Association between RAS-I administration and all-cause mortality

\begin{tabular}{|c|c|c|c|c|c|c|c|c|}
\hline \multirow[b]{2}{*}{ Variable } & \multicolumn{2}{|c|}{ Unadjusted model } & \multicolumn{2}{|c|}{ Model 1} & \multicolumn{2}{|c|}{ Model 2} & \multicolumn{2}{|c|}{ Model 3} \\
\hline & $\begin{array}{r}\text { Crude HR } \\
(95 \% \mathrm{CI}) \\
\end{array}$ & $P$ value & $\begin{array}{l}\text { Adjusted HR } \\
(95 \% \mathrm{CI})\end{array}$ & $P$ value & $\begin{array}{l}\text { Adjusted HR } \\
(95 \% \mathrm{CI})\end{array}$ & $P$ value & $\begin{array}{l}\text { Adjusted HR } \\
(95 \% \mathrm{CI})\end{array}$ & $P$ value \\
\hline \multicolumn{9}{|c|}{$\begin{array}{l}\text { RAS-I } \\
\text { administration }\end{array}$} \\
\hline No & Ref. & Ref. & Ref. & Ref. & Ref. & Ref. & Ref. & Ref. \\
\hline Yes & $\begin{array}{l}1.160 \\
(0.771-1.743)\end{array}$ & 0.477 & $\begin{array}{l}0.845 \\
(0.561-1.272)\end{array}$ & 0.419 & $\begin{array}{l}0.562 \\
(0.371-0.852)\end{array}$ & 0.007 & $\begin{array}{l}0.499 \\
(0.325-0.767)\end{array}$ & 0.002 \\
\hline \multicolumn{9}{|c|}{$\begin{array}{l}\text { ACEI } \\
\text { administration }\end{array}$} \\
\hline No & Ref. & Ref. & Ref. & Ref. & Ref. & Ref. & Ref. & Ref. \\
\hline Yes & $\begin{array}{l}2.767 \\
(1.542-4.967)\end{array}$ & 0.001 & $\begin{array}{l}1.962 \\
(1.091-3.528)\end{array}$ & 0.024 & $\begin{array}{l}0.885 \\
(0.488-1.605)\end{array}$ & 0.688 & $\begin{array}{l}0.892 \\
(0.473-1.682)\end{array}$ & 0.724 \\
\hline \multicolumn{9}{|c|}{$\begin{array}{l}\text { ARB } \\
\text { administration }\end{array}$} \\
\hline No & Ref. & Ref. & Ref. & Ref. & Ref. & Ref. & Ref. & Ref. \\
\hline Yes & $\begin{array}{l}0.775 \\
(0.464-1.295)\end{array}$ & 0.331 & $\begin{array}{l}0.579 \\
(0.346-0.967)\end{array}$ & 0.037 & $\begin{array}{l}0.457 \\
(0.272-0.770)\end{array}$ & 0.003 & $\begin{array}{l}0.410 \\
(0.240-0.700)\end{array}$ & 0.001 \\
\hline
\end{tabular}

Model 1: adjusted for age and gender. Model 2: model 1, hypertension, diabetes, CHD, LYM. Model 3: model 2, anti-virus drugs, glucocorticoid, tocilizumab, chloroquine/ hydroxychloroquine, ARDS, heart failure, AMI, AKI.

Abbreviations: RAS-I, renin-angiotensin inhibitor; ACEI, angiotensin converting enzyme inhibitor; ARB, angiotensin receptor blocker; HR, hazard ratio; CHD, coronary heart disease; LYM, lymphocyte count; ARDS, acute respiratory distress syndrome; AMI, acute myocardial infarction; AKI, acute kidney injury. 
associated with a reduced risk of all-cause mortality among patients with COVID-19 and hypertension. No significant association was found between ACEI use and all-cause mortality $(\mathrm{HR}=1.008,95 \%$ CI 0.231-4.391) (Table 4).

\section{Discussion}

This study revealed the protective effects of RAS-I administration for all-cause mortality among hospitalized patients with COVID-19. ARB use was significantly associated with a reduced risk of mortality among patients with COVID-19. A similar observation was found in the subgroup analyses of patients with hypertension. This work provided evidence supporting RAS-I use, especially ARB use, for patients with COVID-19.
Inconsistent results have been reported regarding the association between RAS-I use and COVID-19 outcome. Mehta et al. analyzed the association between RAS-I use and clinical outcomes among 1735 patients with COVID19 in the United States and reported higher mortality among patients receiving RAS-I $(n=8,3.8 \%)$ than those not receiving RAS-I $(n=34,2.1 \%)$ [13]. In Asia, several relevant studies were conducted among patients with hypertension or diabetes. By collecting data from 1178 hypertensive patients with COVID-19, Li et al. did not observe differences in RAS-I use between non-survivors and survivors $(27.3 \%$ vs. $33.0 \% ; P=0.34)$ [9]. Chen et al. compared the clinical characteristics of patients with diabetes who are receiving or not receiving RAS-I and found no significant difference [21]. Zhang et al. reported a decreased risk for all-cause mortality with an adjusted HR

Table 3 Demographic characteristics among patients with COVID-19 with hypertension

\begin{tabular}{|c|c|c|c|c|}
\hline \multirow{2}{*}{ Factors } & \multirow{2}{*}{ Overall $(n=590)$} & \multicolumn{2}{|c|}{ RAS-I administration } & \multirow{2}{*}{$P$ value } \\
\hline & & Yes $(n=141)$ & No $(n=449)$ & \\
\hline Age, $n(\%)$ & & & & 0.540 \\
\hline$<18$ years & $0(0.0 \%)$ & $0(0.0 \%)$ & $0(0.0 \%)$ & \\
\hline $18-44$ years & $21(3.6 \%)$ & $7(5.0 \%)$ & $14(3.1 \%)$ & \\
\hline 45-64 years & $230(39.0 \%)$ & $55(39.0 \%)$ & $175(39.0 \%)$ & \\
\hline$\geqslant 65$ years & $339(57.5 \%)$ & $79(56.0 \%)$ & $260(57.9 \%)$ & \\
\hline Male, $n(\%)$ & $293(49.7 \%)$ & $68(48.2 \%)$ & $225(50.1 \%)$ & 0.700 \\
\hline \multicolumn{5}{|l|}{ Laboratory test } \\
\hline $\mathrm{WBC}\left(\times 10^{9}\right.$ cells $\left./ \mathrm{L}\right)$ & $7.1(5.6,9.5)$ & $6.4(5.3,9.0)$ & $7.2(5.6,9.5)$ & 0.260 \\
\hline LYM $\left(\times 10^{9}\right.$ cells $\left./ \mathrm{L}\right)$ & $1.1(0.7,1.6)$ & $1.1(0.6,1.5)$ & $1.1(0.7,1.6)$ & 0.690 \\
\hline hs-CRP (mg/L) & $38.0(4.5,102.1)$ & $22.2(4.6,72.3)$ & $42.8(4.5,111.3)$ & 0.980 \\
\hline PCT (ng/L) & $0.09(0.05,0.24)$ & $0.08(0.04,0.23)$ & $0.09(0.05,0.24)$ & 0.340 \\
\hline IL-6 (pg/mL) & $8.3(3.2,15.6)$ & $9.7(3.0,15.5)$ & $8.3(3.3,15.6)$ & 0.790 \\
\hline hs-cTn (ng/mL) & $7.9(3.3,20.6)$ & $8.6(4.1,14.3)$ & $7.8(3.3,20.6)$ & 0.780 \\
\hline $\mathrm{SCr}(\mu \mathrm{mol} / \mathrm{L})$ & $73.4(61.0,92.0)$ & $86.0(69.0,103.0)$ & $73.0(60.1,92.0)$ & 0.100 \\
\hline SBP (mmHg) & $129.7(122.3,137.0)$ & $135.6(124.6,141.9)$ & $129.6(122.3,137.0)$ & 0.220 \\
\hline DBP (mmHg) & $76.6(72.1,82.3)$ & $80.5(74.1,86.2)$ & $76.5(72.1,82.3)$ & 0.180 \\
\hline \multicolumn{5}{|l|}{ Complication, $n(\%)$} \\
\hline Acute respiratory distress syndrome & $84(14.2 \%)$ & $13(9.2 \%)$ & $71(15.8 \%)$ & 0.051 \\
\hline Heart failure & $85(14.4 \%)$ & $18(12.8 \%)$ & $67(14.9 \%)$ & 0.520 \\
\hline Acute myocardial infarction & $98(16.6 \%)$ & $19(13.5 \%)$ & $79(17.6 \%)$ & 0.250 \\
\hline Acute kidney injury & $38(6.4 \%)$ & $8(5.7 \%)$ & $30(6.7 \%)$ & 0.840 \\
\hline Multiple organ dysfunction syndrome & $76(12.9 \%)$ & $12(8.5 \%)$ & $64(14.3 \%)$ & 0.076 \\
\hline Severity, $n(\%)$ & & & & 0.730 \\
\hline Mild cases & $10(1.7 \%)$ & $2(1.4 \%)$ & $8(1.8 \%)$ & \\
\hline Moderate cases & $209(35.4 \%)$ & $53(37.6 \%)$ & $156(34.7 \%)$ & \\
\hline Severe cases & $265(44.9 \%)$ & $65(46.1 \%)$ & $200(44.5 \%)$ & \\
\hline Critical & $106(18.0 \%)$ & $21(14.9 \%)$ & $85(18.9 \%)$ & \\
\hline Outcome, $n(\%)$ & & & & 0.029 \\
\hline Recovery & $526(89.2 \%)$ & $133(94.3 \%)$ & $393(87.5 \%)$ & \\
\hline Death & $64(10.8 \%)$ & $8(5.7 \%)$ & $56(12.5 \%)$ & \\
\hline
\end{tabular}

Abbreviations: RAS-I, renin-angiotensin system inhibitor; WBC, while blood cell; LYM, lymphocyte count; hs-CRP, hyper-sensitive C-reactive protein; PCT, procalcitonin; IL-6, interleukin-6; hs-cTn, hyper-sensitive cardiac troponin; SCr, serum creatinine; SBP, systolic blood pressure; DBP, diastolic blood pressure. 
Table 4 Association between RAS-I administration and all-cause mortality among patients with hypertension

\begin{tabular}{|c|c|c|c|c|c|c|}
\hline \multirow{2}{*}{ Variable } & \multicolumn{2}{|c|}{ Unadjusted model } & \multicolumn{2}{|c|}{ Model 1} & \multicolumn{2}{|c|}{ Model 2} \\
\hline & Crude HR $(95 \% \mathrm{CI})$ & $P$ value & Adjusted HR $(95 \% \mathrm{CI})$ & $P$ value & Adjusted HR $(95 \% \mathrm{CI})$ & $P$ value \\
\hline \multicolumn{7}{|c|}{ RAS-I administration } \\
\hline No & Ref. & Ref. & Ref. & Ref. & Ref. & Ref. \\
\hline Yes & $0.402(0.192-0.845)$ & 0.016 & $0.433(0.206-0.909)$ & 0.027 & $0.352(0.162-0.764)$ & 0.008 \\
\hline \multicolumn{7}{|c|}{ ACEI administration } \\
\hline No & Ref. & Ref. & Ref. & Ref. & Ref. & Ref. \\
\hline Yes & $0.691(0.169-2.824)$ & 0.606 & $0.663(0.162-2.713)$ & 0.567 & $1.008(0.231-4.391)$ & 0.992 \\
\hline \multicolumn{7}{|c|}{ ARB administration } \\
\hline No & Ref. & Ref. & Ref. & Ref. & Ref. & Ref. \\
\hline Yes & $0.353(0.152-0.818)$ & 0.015 & $0.381(0.164-0.884)$ & 0.025 & $0.279(0.115-0.677)$ & 0.005 \\
\hline
\end{tabular}

Model 1: adjusted for age and gender. Model 2: model 1, LYM, anti-virus drugs, glucocorticoid, ARDS, heart failure, AMI, AKI.

Abbreviations: RAS-I, renin-angiotensin system inhibitor; ACEI, angiotensin converting enzyme inhibitor; ARB, angiotensin receptor blocker; HR, hazard ratio; LYM, lymphocyte count; ARDS, acute respiratory distress syndrome; AMI, acute myocardial infarction; AKI, acute kidney injury.

of 0.42 (95\% CI 0.19-0.92) among hypertensive patients with COVID-19 who received RAS-I [22]. However, no stratified results on ACEI and ARB use were provided. By using a relatively large sample size of patients with COVID-19 and after adjusting for extensive covariates, the present study supported the protective effects of RAS-I and ARB on survival outcomes among patients with COVID19 with or without hypertension.

As a major hormonal system, RAS plays pathogenetic and protective roles in complex regulation mechanisms [5,23-25]. In this system, angiotensin I could be converted into angiotensin II by ACE-induced vasoconstriction, inflammation, and fibrosis through the $\mathrm{ACE}-\mathrm{AT}_{1}$ axis; angiotensin II can either directly interact with $\mathrm{AT}_{2}$ receptor or be catalyzed by ACE2 into angiotensin 1-7 and interact with Mas receptor, thus promoting vasodilatation, antiinflammation, and anti-fibrosis [5,24]. ARBs inhibit the risk effects by blocking the $\mathrm{AT}_{1}$ receptor; hence, the counter-regulatory increased angiotensin II could interact with $\mathrm{AT}_{2}$ receptor and be catalyzed into angiotensin 1-7 $[5,24,26]$. Ultimately, the protective effects would be promoted through multiple approaches to maintain physiological homeostasis. The benefits of ARB on the allcause mortality of patients with COVID-19 are speculated to originate from the physiological homeostasis. In theory, ACEI could also inhibit the risk effects of RAS, though the pathway is different from that of ARB [24]. However, this study found no significant association between the use of ACEI and the survival outcome of patients with COVID19. The result might be influenced by the small sample size (43 patients used ACEI). A recent work reported the potentially harmful effects of ACEI on the outcome of patients with COVID-19 and diabetes in Iran [27]. Hence, further research with a large sample size of ACEI users with COVID-19 in different populations is still needed to verify the association between ACEI use and survival outcome.
Hypertension is a risk factor of lower respiratory tract infections and community-acquired pneumonia, especially among the elderly $[28,29]$. High prevalence of hypertension was observed among patients with COVID-19 [2,11], though robust evidence supporting the association between hypertension and COVID-19 risk is lacking. The inflammatory activation in hypertension is its pathogenetic linkage to COVID-19 [24]. Similar lymphocyte loss and cytokine overproduction such as IL-6 were observed in hypertension and COVID-19 [15,30,31]. The activated RAS in hypertension further augmented the lung injury through the angiotensin II-ACE-AT ${ }_{1}$ receptor axis [7,32]. ACEI and ARB could block the angiotensin II-ACE-AT 1 receptor axis, though their target molecules are different [24]. Attenuated inflammation and few ARDS cases were observed among patients with hypertension who received RAS-I. Meng et al. also reported mild inflammation among patients with COVID-19 receiving RAS-I [10]. This phenomenon may partly lead to the beneficial effect of RAS-I administration on all-cause mortality among hypertensive patients.

This study has several limitations. First, given the observational nature of this work and the use of electronic medical records as the basis, the possibility of residual confounding exists. Second, only in-hospital all-cause mortality was documented. Third, due to the relatively low percentage of ACEI use in China, the analyses regarding the association between ACEI and COVID-19 outcome might be underpowered. Finally, the observational design limited the ability to generate causal inference in the analyses. Further prospective randomized trials are needed to reveal the protective effects of RAS-I on the survival outcome of patients with COVID-19.

In conclusion, this work provides evidence that $\mathrm{ARB}$ administration is strongly associated with a reduced risk of in-hospital all-cause mortality among patients with COVID-19 and thus could be preferably considered for 
patients with COVID-19 independent of hypertension comorbidity. Further investigations using large sample sizes and interventional studies are needed to illustrate the effects of ACEI use among patients with COVID-19.

\section{Acknowledgements}

This study was supported by grants from Special Research Fund of PKU for Prevention and Control of COVID-19 and the Fundamental Research Funds for the Central Universities (Nos. PKU2020PKYZX003 and BMU2020HKYZX007), the National Natural Science Foundation of China (Nos. 91846101, 81771938, 81301296, 81900665, 81570667, 81470948, and 81670633), Major Research Plan of the National Natural Science Foundation of China (No. 91742204), The International (Regional) Cooperation and Exchange Projects (NSFC-DFG, No. 81761138041), Beijing Nova Programme Interdisciplinary Cooperation Project (No. Z191100001119008), the National Key R\&D Program of the Ministry of Science and Technology of China (Nos. 2016YFC1305405, 2019YFC2005000, 2018YFC1314003-1, and 2015BAI12B07), National Key Research and Development Program (No. 2016YFC0906103), the University of Michigan Health System-Peking University Health Science Center Joint Institute for Translational and Clinical Research (Nos. BMU20160466, BMU2018JI012, and BMU2019JI005), Beijing Advanced Discipline Construction Project (No. BMU2019GJJXK001), PKU-Baidu Fund (No. 2019BD017) and from Peking University (Nos. BMU2018MX020 and PKU2017LCX05).

\section{Compliance with ethics guidelines}

Huai-yu Wang, Suyuan Peng, Zhanghui Ye, Pengfei Li, Qing Li, Xuanyu Shi, Rui Zeng, Ying Yao, Fan He, Junhua Li, Liu Liu, Shuwang Ge, Xianjun Ke, Zhibin Zhou, Gang Xu, Ming-hui Zhao, Haibo Wang, Luxia Zhang, and Erdan Dong declare no conflict of interests. This study was conducted with the authorization of National Health Commission of the People Republic of China and was approved by the Ethics Committee of Peking University Health Science Center (IRB00001052-20032) .

\section{References}

1. Patel AB, Verma A. COVID-19 and angiotensin-converting enzyme inhibitors and angiotensin receptor blockers: what is the evidence? JAMA 2020; 323(18): 1769-1770

2. Guan WJ, Ni ZY, Hu Y, Liang WH, Ou CQ, He JX, Liu L, Shan H, Lei CL, Hui DSC, Du B, Li LJ, Zeng G, Yuen KY, Chen RC, Tang CL, Wang T, Chen PY, Xiang J, Li SY, Wang JL, Liang ZJ, Peng YX, Wei L, Liu Y, Hu YH, Peng P, Wang JM, Liu JY, Chen Z, Li G, Zheng ZJ, Qiu SQ, Luo J, Ye CJ, Zhu SY, Zhong NS; China Medical Treatment Expert Group for Covid-19. Clinical characteristics of coronavirus disease 2019 in China. N Engl J Med 2020; 382 (18): 1708-1720

3. Zhang H, Penninger JM, Li Y, Zhong N, Slutsky AS. Angiotensinconverting enzyme 2 (ACE2) as a SARS-CoV-2 receptor: molecular mechanisms and potential therapeutic target. Intensive Care Med 2020; 46(4): 586-590

4. Zhou G, Chen S, Chen Z. Advances in COVID-19: the virus, the pathogenesis, and evidence-based control and therapeutic strategies. Front Med 2020; 14(2): 117-125

5. Simões E Silva AC, Teixeira MM. ACE inhibition, ACE2 and angiotensin-(1-7) axis in kidney and cardiac inflammation and fibrosis. Pharmacol Res 2016; 107: 154-162

6. South AM, Diz DI, Chappell MC. COVID-19, ACE2, and the cardiovascular consequences. Am J Physiol Heart Circ Physiol 2020; 318(5): H1084-H1090

7. Imai Y, Kuba K, Rao S, Huan Y, Guo F, Guan B, Yang P, Sarao R, Wada T, Leong-Poi H, Crackower MA, Fukamizu A, Hui CC, Hein L, Uhlig S, Slutsky AS, Jiang C, Penninger JM. Angiotensinconverting enzyme 2 protects from severe acute lung failure. Nature 2005; 436(7047): 112-116

8. South AM, Tomlinson L, Edmonston D, Hiremath S, Sparks MA. Controversies of renin-angiotensin system inhibition during the COVID-19 pandemic. Nat Rev Nephrol 2020; 16(6): 305-307

9. Li J, Wang X, Chen J, Zhang H, Deng A. Association of reninangiotensin system inhibitors with severity or risk of death in patients with hypertension hospitalized for coronavirus disease 2019 (COVID-19) infection in Wuhan, China. JAMA Cardiol 2020; 5(7): 825-830

10. Meng J, Xiao G, Zhang J, He X, Ou M, Bi J, Yang R, Di W, Wang Z, Li Z, Gao H, Liu L, Zhang G. Renin-angiotensin system inhibitors improve the clinical outcomes of COVID-19 patients with hypertension. Emerg Microbes Infect 2020; 9(1): 757-760

11. Reynolds HR, Adhikari S, Pulgarin C, Troxel AB, Iturrate E, Johnson SB, Hausvater A, Newman JD, Berger JS, Bangalore S, Katz SD, Fishman GI, Kunichoff D, Chen Y, Ogedegbe G, Hochman JS. Renin-angiotensin-aldosterone system inhibitors and risk of Covid-19. N Engl J Med 2020; 382(25): 2441-2448

12. Mancia G, Rea F, Ludergnani M, Apolone G, Corrao G. Reninangiotensin-aldosterone system blockers and the risk of Covid-19. N Engl J Med 2020; 382(25): 2431-2440

13. Mehta N, Kalra A, Nowacki AS, Anjewierden S, Han Z, Bhat P, Carmona-Rubio AE, Jacob M, Procop GW, Harrington $S$, Milinovich A, Svensson LG, Jehi L, Young JB, Chung MK. Association of use of angiotensin-converting enzyme inhibitors and angiotensin II receptor blockers with testing positive for coronavirus disease 2019 (COVID-19). JAMA Cardiol 2020; 5(9): 1020-1026

14. Xu J, Huang C, Fan G, Liu Z, Shang L, Zhou F, Wang Y, Yu J, Yang L, Xie K, Huang Z, Huang L, Gu X, Li H, Zhang Y, Wang Y, Hayden FG, Horby PW, Cao B, Wang C. Use of angiotensinconverting enzyme inhibitors and angiotensin II receptor blockers in context of COVID-19 outbreak: a retrospective analysis. Front Med 2020; 14(5): 601-612

15. The National Health Commission of China. Diagnosis and Treatment Protocol for COVID-19 (Trial Version 7). 2020. http:// en.nhc.gov.cn/2020-03/29/c_78469.htm (accessed March 29, 2020)

16. ARDS Definition Task Force, Ranieri VM, Rubenfeld GD, Thompson BT, Ferguson ND, Caldwell E, Fan E, Camporota L, Slutsky AS. Acute respiratory distress syndrome: the Berlin Definition. JAMA 2012; 307(23): 2526-2533

17. Ponikowski P, Voors AA, Anker SD, Bueno H, Cleland JG, Coats AJ, Falk V, González-Juanatey JR, Harjola VP, Jankowska EA, 
Jessup M, Linde C, Nihoyannopoulos P, Parissis JT, Pieske B, Riley JP, Rosano GM, Ruilope LM, Ruschitzka F, Rutten FH, van der Meer P; Authors/Task Force Members; Document Reviewers. 2016 ESC Guidelines for the diagnosis and treatment of acute and chronic heart failure: The Task Force for the diagnosis and treatment of acute and chronic heart failure of the European Society of Cardiology (ESC). Developed with the special contribution of the Heart Failure Association (HFA) of the ESC. Eur J Heart Fail 2016; 18(8): 891975

18. Reichlin T, Twerenbold R, Reiter M, Steuer S, Bassetti S, Balmelli C, Winkler K, Kurz S, Stelzig C, Freese M, Drexler B, Haaf P, Zellweger C, Osswald S, Mueller C. Introduction of high-sensitivity troponin assays: impact on myocardial infarction incidence and prognosis. Am J Med 2012; 125(12): 1205-1213.e1

19. Kidney Disease: Improving Global Outcomes (KDIGO) CKD Work Group. Kidney Disease: Improving Global Outcomes-CKD Evaluation and Management 2012. https://kdigo.org/guidelines/ ckd-evaluation-and-management/ (accessed March 29, 2020)

20. Ng'andu NH. An empirical comparison of statistical tests for assessing the proportional hazards assumption of Cox's model. Stat Med 1997; 16(6): 611-626

21. Chen Y, Yang D, Yang C, Zheng L, Huang K. Response to Comment on Chen et al. Clinical characteristics and outcomes of patients with diabetes and COVID-19 in association with glucoselowering medication. Diabetes Care 2020; 43: 1399-1407. Diabetes Care 2020; 43(10): e165-e166

22. Zhang P, Zhu L, Cai J, Lei F, Qin JJ, Xie J, Liu YM, Zhao YC, Huang X, Lin L, Xia M, Chen MM, Cheng X, Zhang X, Guo D, Peng Y, Ji YX, Chen J, She ZG, Wang Y, Xu Q, Tan R, Wang H, Lin J, Luo P, Fu S, Cai H, Ye P, Xiao B, Mao W, Liu L, Yan Y, Liu M, Chen M, Zhang XJ, Wang X, Touyz RM, Xia J, Zhang BH, Huang X, Yuan Y, Loomba R, Liu PP, Li H. Association of inpatient use of angiotensin-converting enzyme inhibitors and angiotensin II receptor blockers with mortality among patients with hypertension hospitalized with COVID-19. Circ Res 2020; 126(12): 16711681

23. Culver S, Li C, Siragy HM. Intrarenal angiotensin-converting enzyme: the old and the new. Curr Hypertens Rep 2017; 19(10): 80

24. Kreutz R, Algharably EAE, Azizi M, Dobrowolski P, Guzik T, Januszewicz A, Persu A, Prejbisz A, Riemer TG, Wang JG, Burnier
M. Hypertension, the renin-angiotensin system, and the risk of lower respiratory tract infections and lung injury: implications for COVID-19. Cardiovasc Res 2020; 116(10): 1688-1699

25. Anguiano L, Riera M, Pascual J, Soler MJ. Circulating ACE2 in cardiovascular and kidney diseases. Curr Med Chem 2017; 24(30): 3231-3241

26. Eberhardt RT, Kevak RM, Kang PM, Frishman WH. Angiotensin II receptor blockade: an innovative approach to cardiovascular pharmacotherapy. J Clin Pharmacol 1993; 33(11): 1023-1038

27. Aghaaliakbari F, Abbasi MA, Ranjbar M, Jamshidi Makiani M, Farrokhpour M, Safarnezhad Tameshkel F, Karbalaie Niya MH, Doltkhah S, Yaghoobzadeh K, Savaj S. Angiotensin converting enzyme inhibitors, a risk factor of poor outcome in diabetic patients with COVID-19 infection. Iran J Kidney Dis 2020; 14(6): 482487

28. Gutiérrez F, Masiá M, Mirete C, Soldán B, Rodríguez JC, Padilla S, Hernández I, Royo G, Martin-Hidalgo A. The influence of age and gender on the population-based incidence of community-acquired pneumonia caused by different microbial pathogens. J Infect 2006; 53(3): 166-174

29. Woodhead M, Blasi F, Ewig S, Garau J, Huchon G, Ieven M, Ortqvist A, Schaberg T, Torres A, van der Heijden G, Read R, Verheij TJ; Joint Taskforce of the European Respiratory Society and European Society for Clinical Microbiology and Infectious Diseases. Guidelines for the management of adult lower respiratory tract infections - full version. Clin Microbiol Infect 2011; 17(Suppl 6): E1-E59

30. Chou CH, Hung CS, Liao CW, Wei LH, Chen CW, Shun CT, Wen WF, Wan $\mathrm{CH}, \mathrm{Wu} \mathrm{XM}$, Chang YY, Wu VC, Wu KD, Lin YH; TAIPAI Study Group. IL-6 trans-signalling contributes to aldosterone-induced cardiac fibrosis. Cardiovasc Res 2018; 114(5): 690702

31. Siedlinski M, Jozefczuk E, Xu X, Teumer A, Evangelou E, Schnabel RB, Welsh P, Maffia P, Erdmann J, Tomaszewski M, Caulfield MJ, Sattar N, Holmes MV, Guzik TJ. White blood cells and blood pressure: a Mendelian randomization study. Circulation 2020; 141 (16): 1307-1317

32. Imai Y, Kuba K, Penninger JM. The discovery of angiotensinconverting enzyme 2 and its role in acute lung injury in mice. Exp Physiol 2008; 93(5): 543-548 